\title{
Seletividade de acaricidas e inseticidas a ácaros predadores (Acari: Phytoseiidae) encontrados em seringueira no centro-oeste do Brasil
}

\author{
Side-effect of acaricides and insecticides to predatory mites (Acari: Phytoseiidae) found in \\ rubber-trees in mid-west Brazil.
}

\author{
Noeli Juarez Ferla ${ }^{2}$ Gilberto José de Moraes ${ }^{3}$
}

\section{RESUMO}

Euseius concordis (Chant) e Neoseiulus anonymus (Chant \& Baker) são ácaros predadores da família Phytoseiidae comumente encontrados em seringueira (Hevea brasiliensis Muell. Arg.) em Mato Grosso, região centro-oeste do Brasil. Este trabalho foi realizado para verificar o efeito de acaricidas $e$ inseticidas-acaricidas empregados em plantações de seringueira, e outros que teriam potencial para serem empregados contra insetos e ácaros considerados pragas dessa cultura, sobre $\boldsymbol{E}$. concordis $e \mathrm{~N}$. anonymus. Utilizou-se o método residual de pulverização em superfície, recomendado como padrão pelo Grupo de Trabalho "Pesticidas e Artrópodes Benéficos”, da Organização Internacional de Controle Biológico e Integrado de Plantas e Animais Nocivos/Seção Regional do Paleártico Oeste. Duas concentrações de cada um dos seguintes ingredientes ativos foram utilizadas: acefato, dicofol, endosulfan, formetanate, metomil, monocrotofós, óxido de fenbutatin e propargite. Uma das concentrações utilizadas foi uma média daquelas sugeridas pelos fabricantes para o controle de ácaros e insetos fitófagos presentes em outras culturas, uma vez que nenhum dos produtos testados tem registro para o uso em seringueira e a outra correspondeu à cerca de um terço da primeira. Endosulfan a 320ppm, dicofol a 100ppm e óxido de fenbutatin a 100 e 320ppm foram inócuos a $\boldsymbol{E}$. concordis, enquanto que endosulfan a 320ppm e dicofol a 100ppm foram inócuos a N. anonymus. Acefato, formetanate e monocrotofós, nas concentrações testadas, foram nocivos às duas espécies.

Palavras-chave: controle biológico, predador, MIP, Hevea brasiliensis, produtos fitossanitários.

\section{ABSTRACT}

Euseius concordis (Chant) and Neoseiulus anonymus (Chant \& Baker) are predatory mites of the family
Phytoseiidae commonly found on rubber trees (Hevea brasiliensis Muell. Arg.) in the State of Mato Grosso, in the mid-west Brazil. This work was conducted to verify the effect of acaricides and insecticides-acaricides occasionally used in rubber tree plantations, and other products that could be used against different pests (insects and mites) in this crop, on $\boldsymbol{E}$. concordis and $N$. anonymus. The residual contact spray method in glass surface was used, which is recommended by the Working Group on "Pesticides and Benefic Arthropods" of the International Organization of Biologic and Integrated Control of Noxious Plants and Animals/Regional Section of the West Paleartic (IOBC/WPRS). Two concentrations of the following active ingredients were used: acephato, dicofofol, endosulfan, formetanate, metomil, monocrotophos, oxid of fenbutanin and propargit. For each product, one of the concentrations used was an average of the concentrations recommended by the manufacturers for the control of mites and phytophagous insects in other crops, since none of the tested products are registered for use on rubber tree. The other concentration corresponded to ca. a third of the former concentration. Endosulfan at $320 \mathrm{ppm}$, dicofol at 100ppm and fenbutanin oxid at 100 and 320 ppm were harmless to $\boldsymbol{E}$. concordis, while endosulfan at $320 \mathrm{ppm}$ and dicofol at 100ppm were harmless to $\mathrm{N}$. anonymus. Acephate, formetanate and monocrotofós, in concentrations, were harmful to both species.

Key words: biological control, predator, IPM, Hevea brasiliensis, pesticides.

\section{INTRODUÇÃO}

A seringueira (Hevea brasiliensis Muell. Arg.) é comumente atacada por diversas espécies de insetos e ácaros no centro-oeste do Brasil e no Estado de São Paulo, sendo algumas espécies acarinas consideradas pragas (FERES, 2000). Apesar da cultura

${ }^{1}$ Departamento de Entomologia, Fitopatologia e Zoologia Agrícola, Escola Superior de Agricultura “Luiz de Queiroz” (ESALQ), Universidade de São Paulo (USP), Piracicaba, São Paulo, Brasil

${ }^{2}$ Centro Universitário UNIVATES. Rua Avelino Tallini, 171, CP 155, 95900-000, Lajeado, Rio Grande do Sul, Brasil. E-mail: njferla@univates.br

${ }^{3}$ Departamento de Entomologia, Fitopatologia e Zoologia Agrícola, ESALQ, USP, Piracicaba, São Paulo, Brasil. 
da seringueira ser nativa e explorada economicamente na região amazônica desde o século XVIII, pouco se sabe sobre os ácaros presentes nesta cultura. A maioria dos trabalhos realizados, até o momento, se refere à descrição de novas espécies ou relata a presença de espécies atacando-a. Muito pouco é conhecido sobre a flutuação populacional ou o papel desempenhado pelos ácaros em seringueira.

Os ácaros passaram a ser considerados economicamente importantes a partir dos anos 90, quando foram responsabilizados por danos causados a esta cultura e pela conseqüente redução de produtividade. Os ácaros fitófagos mais importantes na cultura da seringueira pertencem às famílias Eriophyidae, Tenuipalpidae e Tetranychidae (FAZOLIN \& PEREIRA, 1989; FLECHTMANN, 1989; FERES, 2000). Dentre estes, Calacarus heveae Feres e Tenuipalpus heveae Baker são considerados os mais importantes (FERES, 1992; FERES, 2000; FERLA \& MORAES, 2002). Produtores da região noroeste do Estado de São Paulo estimam perda de até 30\% na produção de látex de algumas variedades de $\boldsymbol{H}$. brasiliensis pelo ataque intenso de $\boldsymbol{C}$. heveae (FERES, 2000). Segundo VIEIRA \& GOMES (1999), esta espécie pode provocar níveis de desfolha acima de $75 \%$ em clone da variedade RRIM 600.

Ácaros Phytoseiidae e Stigmaeidae são os predadores mais comuns em folhas de seringueira no sudoeste e centro-oeste do Brasil, sendo a maior diversidade apresentada pelos fitoseídeos. Duas espécies de fitoseídeos têm sido freqüentemente encontradas no Estado de Mato Grosso, Euseius concordis (Chant) e Neoseiulus anonymus (Chant \& Baker) (FERLA \& MORAES, 2002). De acordo com resultados observados por FERLA \& MORAES (2003a), $N$. anonymus parece ser uma espécie importante no controle de tetraniquídeos nesta cultura, enquanto que E. concordis demonstra preferência por pólen.

Nenhum agroquímico é registrado para o controle de insetos e ácaros na cultura da seringueira. Até o momento, não foram realizados estudos no sentido de conhecer os produtos que apresentam seletividade às espécies de ácaros predadores mais comuns nesta cultura.

Este trabalho foi realizado para verificar o efeito de produtos fitossanitários que são empregados na plantação da seringueira e outros, que teriam potencial para serem usados contra insetos e ácaros pragas da cultura, sobre $\boldsymbol{E}$. concordis e $N$. anonymus.

\section{MATERIAL E MÉTODOS}

O trabalho foi realizado no laboratório da Escola Superior de Agricultura "Luiz de Queiroz”
(ESALQ/USP), em Piracicaba, São Paulo, de novembro de 1999 a fevereiro de 2000, utilizando-se ácaros provenientes de colônias de laboratório iniciadas com indivíduos coletados de folhas de seringueira cerca de dois meses antes do início do trabalho. $\boldsymbol{E}$. concordis foi coletado em Itiquira e $\boldsymbol{N}$. anonymus, em Pontes e Lacerda, ambas no Estado de Mato Grosso.

As colônias de manutenção de ambos os predadores foram mantidas em unidades de criação semelhantes às descritas por MCMURTRY \& SCRIVEN (1965). Cada unidade constou de uma placa de Paviflex ${ }^{\circledR}$ colocada sobre esponja de náilon umedecida com água destilada no interior de uma bandeja plástica, permanecendo totalmente coberta com um pedaço de papelão para evitar a incidência direta da luz sobre os ácaros. Os ácaros foram alimentados com grãos de pólen de taboa (Typha angustifolia L.) colocados sobre lamínulas de microscopia postas na unidade de criação e com uma mistura de diferentes estádios do ácarorajado (Tetranychus urticae Koch) oferecidos a cada dois dias, sobre pedaços de folhas de feijão-de-porco (Canavalia ensiformis (L.) D.C.). A cada dois dias as lamínulas e os pedaços de folha foram substituídos por outros com alimento novo.

Oito acaricidas e inseticidas-acaricidas foram testados (Tabela 1), em duas concentrações, incluindose também um tratamento testemunha, que consistiu da aplicação de água destilada. Cada tratamento teve cinco repetições, em um delineamento experimental inteiramente casualizado. Uma das concentrações utilizadas foi a média daquelas sugeridas pelos fabricantes para o controle de ácaros e insetos fitófagos presentes em outras culturas, de vez que nenhum dos produtos testados tem registro para o uso em seringueira. Outra concentração de cada produto correspondeu a aproximadamente um terço da primeira concentração.

Utilizou-se o método de resíduo de pulverização em uma superfície, recomendado como padrão pelo Grupo de Trabalho "Pesticidas e Artrópodes Benéficos”, da Organização Internacional de Controle Biológico e Integrado de Plantas e Animais Nocivos/Seção Regional do Paleártico Oeste para testar efeitos adversos dos produtos sobre os ácaros predadores(OVERMEER \& VAN ZON, 1982; HASSAN et al., 1985; OVERMEER, 1988; HASSAN et al., 1994).

Discos de dois cm de diâmetro foram recortados de folhas de seringueira para serem usados como superfície para aplicação dos produtos e suporte para os ácaros durante os testes. Estes discos foram levados até a torre de Potter dentro de uma placa de Petri com esponja umedecida e colocados sobre papel toalha mantido sobre o "prato" da torre, para a aplicação do produto. 
Tabela 1 - Acaricidas e inseticidas testados quanto ao efeito sobre E. concordis e N. anonymus. Piracicaba. 1999-2000.

\begin{tabular}{|c|c|c|c|c|c|c|}
\hline Nome técnico & Formulação & Concentração (\%) & Classe toxicológica $^{1}$ & Grupo químico & Dosagem (ppm) & $\mathrm{Uso}^{2}$ \\
\hline acefato & PS & 75 & III & Organofosforado & 180 e 560 & A, I \\
\hline dicofol & $\mathrm{CE}$ & 48 & II & Organoclorado & 100 e 360 & A \\
\hline endosulfan & CE & 35 & I & $\begin{array}{l}\text { Éster do ácido } \\
\text { sulfuroso de diol cíclico }\end{array}$ & 320 e 1000 & A, I \\
\hline formetanate & PS & 50 & I & Carbamato & 32 e 100 & A, I \\
\hline metomil & $\mathrm{CE}$ & 21,5 & I & Carbamato & 90 e 280 & I \\
\hline monocrotofós & $\mathrm{CE}$ & 40 & I & Organofosforado & 560 e 1800 & A, I \\
\hline oxido de fenbutatin & SC & 50 & III & Organoestânico & 100 e 320 & A \\
\hline Propargite & $\mathrm{CE}$ & 72 & II & Fenoxiciclohexil & 320 e 750 & A \\
\hline
\end{tabular}

${ }^{1}$ Classe de toxicidade (Portaria Interministerial MA/MS no 220 de 14/03/1979) I = altamente tóxico; II - medianamente tóxico e III - pouco tóxico.

${ }^{2} \mathrm{~A}=$ Acaricida; $\mathrm{I}$ = Inseticida

Para a pulverização dos produtos, foi utilizada uma torre de Potter para a deposição de 1,5mg do produto $\mathrm{cm}^{-2}$, à pressão de 10 libras pol${ }^{-2}$. Antes da pulverização de cada produto ou água destilada, a torre foi cuidadosamente lavada com água destilada, álcool e acetona. Os primeiros três jatos de cada produto foram sempre eliminados, reduzindo a possibilidade de contaminação com o produto anteriormente utilizado.

Após a aplicação, os discos foram postos a secar em condições ambientais de laboratório em uma placa de Petri por cerca de uma hora, e posteriormente colocados sobre a água presente no interior de uma placa de Petri plástica, sem tampa, de 3,5cm de diâmetro $\mathrm{x} 1 \mathrm{~cm}$ de profundidade, em câmara climatizada $\left(25 \pm 1^{\circ} \mathrm{C}\right)$, fotofase de 12 horas e umidade relativa de $80 \pm 5 \%$. O círculo permaneceu no centro da placa, servindo a água para ser ingerida pelos ácaros e como barreira à sua fuga. Cinco fêmeas aparentemente sadias foram tomadas ao acaso, com pincel e transferidas da unidade de criação para cada círculo tratado. E. concordis foi alimentado com pólen de $\boldsymbol{T}$. angustifolia e $\boldsymbol{N}$. anonymus com exemplares de todos os estádios de Tetranychus mexicanus (McGregor), colocados sobre os discos.

Cada teste teve duração de oito dias, contando-se e retirando-se diariamente as fêmeas mortas. Os discos sobre os quais os ácaros foram mantidos não foram trocados durante a realização do teste. Os ovos postos foram transferidos com pincel das arenas tratadas para outros discos, igualmente tratados, onde foram mantidos para avaliação de viabilidade.

O efeito adverso total foi calculado levandose em conta a mortalidade das fêmeas no tratamento, corrigida em função da mortalidade na testemunha, e o efeito na reprodução (OVERMEER \& VAN ZON, 1982; HASSAN et al., 1985; OVERMEER, 1988; BAKKER et al., 1992). Foram considerados válidos somente os testes em que a mortalidade na testemunha foi de no máximo 20\% (BAKKER et al., 1992). A fórmula utilizada para o cálculo do efeito total foi $E=100 \%-\left(100 \%-M_{C}\right)$ $\mathrm{xE}_{\mathrm{r}}$, na qual $\mathrm{E}=$ efeito total, $\mathrm{M}_{\mathrm{C}}=$ mortalidade corrigida (ABBOTT, 1925) e $E_{r}=$ efeito na reprodução. O efeito na reprodução foi calculado pela divisão da oviposição das fêmeas em cada tratamento pela oviposição média na testemunha $\left(\mathrm{E}_{\mathrm{r}}=\mathrm{R}\right.$ tratamento $\left.\mathrm{R}_{\text {testemunha }}\right)$. $\mathrm{A}$ oviposição média por fêmea $(\mathrm{R})$ foi obtida através da relação: $\mathrm{R}$ = total de ovos viáveis/número de fêmeas vivas no final do teste.

O valor do efeito total obtido para cada produto foi classificado em uma escala de 1 a 4 , conforme critérios estabelecidos pela IOBC/WPRS (BAKKER et al., 1992; HASSAN et al., 1994), sendo classe 1: $\mathrm{E}<30 \%$ (inócuo, não nocivo); classe 2: $30 \%<$ $\mathrm{E}<79 \%$ (levemente nocivo); classe 3: 80\% $<\mathrm{E}<99 \%$ (moderadamente nocivo) e classe 4: $\mathrm{E}>99 \%$ (nocivo).

Os resultados obtidos para a mortalidade foram submetidos a análise de variância e as médias comparadas pelo teste de Tukey a 5\% de probabilidade de erro.

\section{RESULTADOS E DISCUSSÃO}

Na tabela 2, é apresentada a mortalidade corrigida de $\boldsymbol{E}$. concordis e $\boldsymbol{N}$. anonymus nas diferentes concentrações dos acaricidas e inseticidas-acaricidas usados. Dicofol, endosulfan e óxido de fenbutatin causaram baixa mortalidade de $\boldsymbol{E}$. concordis nas menores concentrações usadas. Entretanto, nas maiores concentrações, apenas óxido de fenbutatin apresentou baixa mortalidade desta espécie. Todos os demais produtos testados provocaram mortalidade acima de $80 \%$ para esta espécie. Assim como para $\boldsymbol{E}$. 
Tabela 2 - Toxicidade de acaricidas e inseticidas-acaricidas a fêmeas adultas de $\boldsymbol{E}$. concordis e $\boldsymbol{N}$. anonymus em teste residual de laboratório em diferentes concentrações, 8 dias após o contato $\left(25 \pm 1^{\circ} \mathrm{C}\right.$, fotofase de 12 horas e umidade relativa de $\left.80 \pm 5 \%\right)$. Piracicaba. 1999 2000 .

\begin{tabular}{|c|c|c|c|c|c|c|c|c|c|}
\hline \multirow[t]{2}{*}{ Nome técnico } & \multirow{2}{*}{$\begin{array}{l}\text { Dosagem } \\
\text { (ppm) }\end{array}$} & \multicolumn{2}{|c|}{$\mathrm{M}_{\mathrm{c}}{ }^{1}(\%)$} & \multicolumn{2}{|c|}{$\mathrm{Er}^{2}$} & \multicolumn{2}{|c|}{$E^{3}(\%)$} & \multicolumn{2}{|c|}{ Classe $^{4}$} \\
\hline & & $\begin{array}{c}\text { E. } \\
\text { concordis }\end{array}$ & $\begin{array}{c}N . \\
\text { anonymus }\end{array}$ & $\begin{array}{c}\text { E. } \\
\text { concordis }\end{array}$ & $\begin{array}{c}N . \\
\text { anonymus }\end{array}$ & $\begin{array}{c}E . \\
\text { concordis }\end{array}$ & $\begin{array}{c}N . \\
\text { anonymus }\end{array}$ & $\begin{array}{c}\text { E. } \\
\text { concordis }\end{array}$ & $\begin{array}{c}N . \\
\text { anonymus }\end{array}$ \\
\hline acefato & 180 & $100,0 \mathrm{a}$ & $100,0 \mathrm{a}$ & 0,0 & 0,0 & 100,0 & 100,0 & 4 & 4 \\
\hline acefato & 560 & $100,0 \mathrm{a}$ & 100,0 a & 0,0 & 0,0 & 100,0 & 100,0 & 4 & 4 \\
\hline dicofol & 100 & $33,3 \mathrm{~cd}$ & $34,2 \mathrm{~cd}$ & 1,8 & 1,8 & $-24,3$ & $-40,7$ & 1 & 1 \\
\hline dicofol & 360 & $100,0 \mathrm{a}$ & $75,3 \mathrm{ab}$ & 0,0 & 1,1 & 100,0 & 60,6 & 4 & 2 \\
\hline endosulfan & 320 & 42,8 bcd & $4,1 \mathrm{~d}$ & 1,2 & 1,6 & 17,8 & $-54,2$ & 1 & 1 \\
\hline endosulfan & 1000 & 88,8 a & 71,7 abc & 1,1 & 0,4 & 100,0 & 80,9 & 4 & 3 \\
\hline formetanate & 32 & $100,0 \mathrm{a}$ & $96,2 \mathrm{a}$ & 0,0 & 0,4 & 100,0 & 100,0 & 4 & 4 \\
\hline formetanate & 100 & $100,0 \mathrm{a}$ & $100,0 \mathrm{a}$ & 0,0 & 0,0 & 100,0 & 100,0 & 4 & 4 \\
\hline metomil & 90 & $100,0 \mathrm{a}$ & 100,0 a & 0,0 & 0,0 & 100,0 & 100,0 & 4 & 4 \\
\hline metomil & 280 & 100,0 a & 92,1 a & 0,0 & 1,3 & 100,0 & 80,6 & 4 & 3 \\
\hline monocrotofós & 560 & $100,0 \mathrm{a}$ & $100,0 \mathrm{a}$ & 0,0 & 0,0 & 100,0 & 100,0 & 4 & 4 \\
\hline monocrotofós & 1800 & 100,0 a & 100,0 a & 0,0 & 0,0 & 100,0 & 100,0 & 4 & 4 \\
\hline $\begin{array}{l}\text { óxido de } \\
\text { fenbutatin }\end{array}$ & 100 & $20,6 \mathrm{~d}$ & 41,5 bcd & 2,1 & 0,8 & 7,3 & 57,0 & 1 & 2 \\
\hline $\begin{array}{l}\text { óxido de } \\
\text { fenbutatin }\end{array}$ & 320 & $10,2 \mathrm{~d}$ & 76,9 a & 1,1 & 1,4 & $-61,7$ & 65,2 & 1 & 2 \\
\hline propargite & 320 & 80,0 a & 90,2 a & 0,8 & 2,3 & 100,0 & 53,5 & 4 & 2 \\
\hline propargite & 750 & $100,0 \mathrm{a}$ & $77,1 \mathrm{ab}$ & 0,0 & 0,6 & 100,0 & 85,8 & 4 & 3 \\
\hline Média & & 79,73 & 78,70 & & & & & & \\
\hline CV (\%) & & 40,15 & 35,82 & & & & & & \\
\hline
\end{tabular}

1. Mortalidade corrigida. Para cada espécie, médias não seguidas da mesma letra diferem estatisticamente entre si, pelo teste de Tukey, a 5\% de probabilidade de erro.

2. Efeito sobre a reprodução.

3. Efeito adverso total.

4. Escala da IOBC/WPRS: classe 1 = inócuo (<30\%); classe 2 = levemente nocivo (30-79\%); classe 3 = moderadamente nocivo (80-99\%); classe 4 = nocivo $(>99 \%)$.

concordis, dicofol, endosulfan e óxido de fenbutatin apresentaram baixa mortalidade de $N$. anonymus nas menores concentrações testadas. Nas menores concentrações dos outros produtos e nas maiores concentrações de todos os produtos testados, a mortalidade de $N$. anonymus foi sempre maior que $70 \%$. Óxido de fenbutatin e endosulfan, nas duas concentrações, e dicofol e propargite, na menor concentração, não tiveram efeito adverso sobre a oviposição de $\boldsymbol{E}$. concordis (Tabela 2). Nas duas concentrações usadas, dicofol não teve efeito adverso na oviposição de $\boldsymbol{N}$. anonymus. As menores concentrações de endosulfan e propargite e as maiores concentrações de metomil e óxido de fenbutatin também não tiveram efeito adverso sobre a oviposição de $N$. anonymus. Efeito negativo sobre a oviposição de $\boldsymbol{N}$. anonymus foi verificado nos tratamentos de formetanate, metomil, óxido de fenbutatin, nas menores concentrações, e endosulfan e propargite, nas maiores concentrações.

Os produtos e/ou concentrações não mencionadas causaram mortalidade total de $\boldsymbol{E}$. concordis e $\boldsymbol{N}$. anonymus, e por isso nenhuma oviposição foi observada nestes casos. Óxido de fenbutatin, na maior concentração, e dicofol, à menor concentração, não tiveram efeito total adverso sobre E. concordis. Endosulfan e óxido de fenbutatin, nas menores concentrações, tiveram pequeno efeito adverso sobre esta espécie. Todos os demais produtos e ou concentrações tiveram efeito altamente adverso sobre $\boldsymbol{E}$. concordis (Tabela 2).

Endosulfan e dicofol, nas menores concentrações, não tiveram efeito total adverso sobre $\boldsymbol{N}$. anonymus. Óxido de fenbutatin, nas duas concentrações, dicofol, na maior concentração, e propargite, na menor concentração, tiveram efeito

Ciência Rural, v.36, n.2, mar-abr, 2006. 
adverso mediano sobre $\boldsymbol{N}$. anonymus. Os demais produtos e ou concentrações tiveram efeito altamente adverso sobre esta espécie.

A maioria dos produtos testados, cerca de $60 \%$, apresentou efeito nocivo aos predadores, sendo que de maneira geral $\boldsymbol{E}$. concordis foi pouco mais suscetível que $\boldsymbol{N}$. anonymus aos produtos. Acefato, formetanate e monocrotofós, nas duas concentrações usadas, foram nocivos às duas espécies.

$\mathrm{O}$ aumento das concentrações conferiu maior toxicidade dos produtos aos predadores, com exceção do óxido de fenbutatin que foi inócuo a $\boldsymbol{E}$. concordis e levemente nocivo a $N$. anonymus nas duas concentrações. Além deste, apenas metomil foi moderadamente nocivo a $\boldsymbol{N}$. anonymus na maior concentração. Dicofol foi inócuo às duas espécies na menor concentração.

Os resultados obtidos, neste estudo, podem subsidiar a escolha dos acaricidas e inseticidas a serem utilizados em programas de manejo integrado na cultura da seringueira que procuram preservar os ácaros predadores $\boldsymbol{E}$. concordis e $\boldsymbol{N}$. anonymus. Estas foram as espécies mais comumente encontradas em avaliações realizadas em plantações de seringueira de Itiquira e Pontes e Lacerda, MT (FERLA \& MORAES, 2002). A manutenção dos inimigos naturais no ambiente pode propiciar uma maior pressão de controle sobre as populações dos ácaros fitófagos sobre as quais aqueles predadores atuam.

A maioria dos acaricidas e inseticidas usados não se mostrou seletiva a $\boldsymbol{E}$. concordis e $\boldsymbol{N}$. anonymus. Apenas dicofol e endosulfan, em baixas concentrações, e óxido de fenbutatin, nas duas concentrações, demonstraram ser seletivos aos predadores. Óxido de fenbutatin também foi considerado inócuo a espécies de fitoseídeos por vários autores, como: OVERMEER \& VAN ZON (1981); STREIBERT (1981); HASSAN et al. (1983); SAMSOEPETERSEN (1983); HASSAN et al. (1987); MORAES et al. (1987); OOMEN et al. (1991) e REIS et al. (1998) e de baixa toxicidade por TANIGOSHI \& FARGERLUND (1984) e HOY \& CONLEY (1987).

Óxido de fenbutatin apresentou efeito negativo à menor concentração e efeito positivo à maior concentração sobre $\boldsymbol{E}$. concordis. Estes resultados possivelmente se devam a alterações fisiológicas nos predadores que apresentaram maior oviposição, quando em presença de maiores concentrações deste produto.

O efeito de acefato, dicofol, endosulfan, formetanate, metomil, monocrotofós, óxido de fenbutatin e propargite, nas mesmas concentrações, sobre $C$. heveae e $T$. heveae, ácaros fitófagos considerados mais importantes na cultura da seringueira, mostrou que $\boldsymbol{C}$. heveae é mais sensível a uma amplitude maior de acaricidas e inseticidasacaricidas que T. heveae. Dicofol e endosulfan, nas menores concentrações, e óxido de fenbutatin, nas duas concentrações, demonstraram ser eficientes no controle de $\boldsymbol{C}$. heveae (FERLA \& MORAES 2003b). Este fato, juntamente com a seletividade aos ácaros predadores em laboratório, indica estes produtos como promissores para a utilização na cultura da seringueira. Entretanto, dicofol e endosulfan são clorados e com registro limitado para poucas culturas. Este fato sugere a necessidade de pesquisar novos ingredientes ativos, mais modernos, que possam ser eficientes e seletivos.

Alguns produtos selecionados, neste estudo, são usados há algum tempo na agricultura e foram escolhidos por serem utilizados no controle do percevejo-de-renda na cultura da seringueira. Desta forma, foi possível avaliar o impacto destes produtos sobre os inimigos naturais de ácaros praga nesta cultura, quando é feito o controle do percevejo-derenda.

\section{CONCLUSÕES}

Os pesticidas Dicofol e Endosulfan, nas concentrações de 100 e 320ppm, respectivamente, e Óxido Fenbutatin, nas concentrações de 100 e 320ppm, são seletivos a $\boldsymbol{E}$. concordis e $\boldsymbol{N}$. anonymus.

\section{AGRADECIMENTOS}

Às empresas Plantações Edouard Michellin Ltda. e Triângulo Agro-Industrial Ltda. pelo financiamento do projeto. Ao Eng. Agrôn. Caio Franchechi e ao Téc. Agr. Nilson de Souza e aos Eng. Agr. Cássio Scomparin e Etiéne Grallien pelo envio dos ácaros fitoseídeos usados nos estudos.

Ao Conselho Nacional de Desenvolvimento Científico e Tecnológico (CNPq), pela concessão de bolsa para a realização do doutorado do primeiro autor.

\section{REFERÊNCIAS}

ABBOTT, W.S. A method of computing the effectiveness of an insecticide. Journal of Economic Entomology, v.18, p.265-267, 1925 .

BAKKER, F.M. et al. Side-effect test for phytoseiids and their rearing methods. IOBC/WPRS Bulletin, v.15, n.3, p.6181, 1992.

FAZOLIN, M.; PEREIRA L.V. Ocorrência de Oligonychus gossypii (Zacher, 1920) (Acari: Tetranychidae) em seringueiras cultivadas. Anais da Sociedade Entomológica do Brasil, v.18, n.1, p.199-202, 1989.

FERES, R.J.F. A new species of Calacarus Keifer (Acari, Eriophyidae, Phyllocoptinae) from Hevea brasiliensis Muell. 
Arg. (Euphorbiaceae) from Brazil. International Journal of Acarology, v.8, n.1, p.61-65, 1992.

FERES, R.J.F. Levantamento e observações naturalísticas da acarofauna (Acari, Arachnida) de seringueiras cultivadas (Hevea spp., Euphorbiaceae) no Brasil. Revista Brasileira de Zoologia, v.17, n.1, p.157-173, 2000.

FLECHTMANN, C.H.W. Seringueira (Hevea sp.) um novo hospedeiro para Oligonychus gossypii (Zacher, 1920) (Acari: Tetranychidae). Boletim do Museu Paraense Emílio Goeldi, v.5, n.1, p.127-128, 1989.

FERLA, N.J.; MORAES, G.J. de. Ácaros (Arachnida, Acari) da seringueira (Hevea brasiliensis Muell. Arg.) no Estado do Mato Grosso, Brasil. Revista Brasileira de Zoologia, v.19, n.3, p.867-888, 2002.

FERLA, N.J.; MORAES, G.J. de. Oviposição dos ácaros predadores Agistemus floridanus Gonzalez, Eusieus concordis (Chant) e Neoseiulus anonymus (Chant \& Baker) (Acari) em resposta a diferentes tipos de alimento. Revista Brasileira de Zoologia, v.20, n.1, p.153-155, $2003 a$.

FERLA, N.J.; MORAES, G.J. de. Efeito de diferentes concentrações de acaricidas e inseticidas-acaricidas sobre Calacarus heveae Feres, 1992 e Tenuipalpus heveae Baker, 1945 (Acari: Eriophyidae e Tenuipalpidae). Acta Biologica Leopoldensia, v.25, n.2, p.179-185, 2003b.

HASSAN, S.A. et al. Results of the second joint pesticide testing programme by the IOBC/WPRS - Working Group "Pesticides and Beneficial Arthropods". Zeitschrift für Angewandte Entomologie, v.95, p.151-158, 1983.

HASSAN, S.A. et al. Standard method to test the side-effects of pesticides on natural enemies of insects and mites developed by the IOBC/WPRS Working Group "Pesticides and Beneficial Organisms”. Bulletin OEPP, v.15, p.214-255, 1985.

HASSAN, S.A. et al. Results of the third joint pesticide testing program by the IOBC/WPRS - Working Group "Pesticides and beneficial Organisms". Journal of Applied Entomology, v.103, p.92-107, 1987.

HASSAN, S.A. et al. Results of the sixth joint pesticide testing program of the IOBC/WPRS - Working Group "Pesticides and Beneficial Organisms”. Entomophaga, v.39, n.1, p.107-119, 1994.

HOY, M.A.; CONLEY. J. Toxicity of pesticides to the western predatory mite. California Agriculture, v.41, n.7/8, p.1214, 1987.
MCMURTRY, J.A.; SCRIVEN. G.T. Insectary production of phytoseiid mites. Journal of Economic Entomology, v.58, n.2, p.282-284, 1965.

MORAES, L.A.H. de, et al. Controle químico do ácaro da ferrugem (Phyllocoptruta oleivora) (Asmead, 1879) (Acari, Eriophyidae) dos citros. Agronomia Sulriograndense, v.23, n.2, p.193-202, 1987.

OVERMEER, W.P.J. Laboratory method for testing side-effects of pesticides on the predacious mites Typhodromus pyri and Amblyseius potentillae (Acari: Phytoseiidae). IOBC/WPRS Bulletin, v.11, n.4, p.65-69, 1988.

OVERMEER, W.P.J.; VAN ZON. A.Q. A comparative study of the effects of some pesticides on three predaceous mite species: Typhodromus pyri, Amblyseius potentillae and A. bibens (Acarina: Phytoseiidae). Entomophaga, v.26, n.10, p.3-9, 1981.

OVERMEER, W.P.J.; VAN ZON. A.Q. A standardized method for testing the side-effects of pesticides on the predaceous mite Amblyseius potentillae (Acarina: Phytoseiidae). Entomophaga, v.27, n.4, p.357-364, 1982.

OOMEN, P.A. et al. Side-effects of 100 pesticides on the predatory mite Phytoseiulus persimilis, collected and evaluated according to the EPPO guideline. Bulletin EPPO, v.21, p.701-712, 1991.

REIS, P.R.L. et al. Seletividade de agroquímicos ao ácaro predador Iphiseiodes zuluagai Denmark \& Muma (Acari: Phytoseiidae). Anais da Sociedade Entomológica do Brasil, v.27, n.2, p.65-74, 1998.

SAMSOE-PETERSEN, L. Laboratory method for testing side effect of pesticides on juvenile stages of the predatory mite, Phytoseiulus persimilis (Acarina: Phytoseiidae) based on detached bean leaves. Entomophaga, v.28, n.2, p.167-178, 1983.

STREIBERT, H.P. A standardized laboratory rearing and testing method for effects pesticides on predatory mite Amblyseius fallacis (Garman). Zeitschrift für Angewandte Entomologie, v.92, p.121-127, 1981.

TANIGOSHI, L.K.; FARGERLUND, J. Implications of parathion resistance of Euseius hibisci (Chant) (Acarina: Phytoseiidae) from the San Joaquin Valley of California. Journal of Economic Entomology, v.77, n.3, p.789-793, 1984.

VIEIRA, M.R.; GOMES, E.C. Sintomas, desfolhamento e controle de Calacarus heveae Feres 1992 (Acari: Eriophyidae) em seringueira (Hevea brasiliensis Muell. Arg.). Cultura Agronômica, v.8, n.1, p.53-71, 1999. 